\title{
Cosmic Ray Signatures from Decaying Gravitino Dark Matter
}

\author{
N.-E. Bomark* \\ University of Bergen, Norway \\ E-mail: nils-erik.bomark@ift.uib.no \\ S. Lola \\ University of Patras, Greece \\ P. Osland \\ University of Bergen, Norway
}

\section{A.R. Raklev}

University of Cambridge, $U K$

\begin{abstract}
We study the charged cosmic rays arising from the slow decay of gravitino dark matter within supersymmetric scenarios with trilinear R-parity violation. It is shown that operators of the $L L \bar{E}$ type can very well account for the recent anomalies in cosmic ray electron and positron data reported by PAMELA, ATIC and Fermi LAT, without violating any other bounds. This scenario will soon be tested by the Fermi LAT data on diffuse gamma ray emission.
\end{abstract}

European Physical Society Europhysics Conference on High Energy Physics

July 16-22, 2009

Krakow, Poland

${ }^{*}$ Speaker. 


\section{Introduction}

Among all dark matter candidates, the most studied ones are clearly the WIMPs, which are particles with electroweak scale masses and annihilation cross sections. In supersymmetric models, the lightest supersymmetric particle (LSP) is a prominent dark matter candidate when it is protected from decay via R-parity, a symmetry first introduced to guarantee the stability of the proton. However, other symmetries could also provide such protection.

Attempts at detecting dark matter have focused on WIMP properties, both in direct detection experiments and when using indirect methods, i.e. the search for annihilation products. At the same time there is no direct empirical evidence in favour of the WIMPs; in fact, we know little about the interaction strength of dark matter.

Gravitinos as dark matter have very different features compared to conventional WIMPs, such as the neutralino, since they do not annihilate at any measurable rate and are not expected to show up in direct detection experiments at all. In fact, gravitino dark matter does not require R-parity; it has been shown that even if this symmetry is violated, the lifetime of the gravitino can easily be larger than the age of the universe [1].

We will here report on a study [2] of supersymmetric models with a gravitino LSP and trilinear R-parity violating operators (for bilinear R-parity violation see [3]):

$$
\lambda L_{i} L_{j} \bar{E}_{k}+\lambda^{\prime} L_{i} Q_{j} \bar{D}_{k}+\lambda^{\prime \prime} \bar{U}_{i} \bar{D}_{j} \bar{D}_{k}
$$

where $L(Q)$ are the left-handed lepton (quark) doublet superfields, and $\bar{E}(\bar{D}, \bar{U})$ are the corresponding left-handed singlet fields. Earlier work [4] studied the gamma radiation from gravitino decays induced by the operators of Eq. (1.1). This is now extended to include charged cosmic rays.

\section{Charged Particles}

Recently, several anomalies in the data on cosmic ray electrons and positrons have been reported. PAMELA has reported an anomalous rise in the positron fraction above $10 \mathrm{GeV}$ [5] and ATIC [6] as well as Fermi LAT [7] have reported an excess in electrons plus positrons at around $100-800 \mathrm{GeV}$. It is believed that these anomalies require a new source of high energy electrons and positrons, and the most commonly discussed possibilities are pulsars and dark matter [8]. Our aim is to see how well these anomalies can be fitted with the decay products from gravitino dark matter, with an emphasis on the Fermi LAT and PAMELA data.

In order to study the cosmic ray flux expected in experiments, we use PYTHIA 6.4 [9] to calculate the spectra of the decay products. We then let GALPROP [10] propagate the particles through the galaxy and calculate the expected background. The GALPROP model we use is a conventional diffusion model where we have rescaled the resulting primary electrons by a factor 0.75 to make room for a simultaneous fit to PAMELA and Fermi LAT. For the dark matter halo density, we assume a NFW profile [11] with parameters $r_{c}=20 \mathrm{kpc}$ and $\rho_{0}=0.33 \mathrm{GeV} \mathrm{cm}^{-3}$.

Operators giving rise to jets, i.e. $L Q \bar{D}$ and $\bar{U} \bar{D} \bar{D}$ operators, will produce large numbers of electrons and positrons through charged pions. The resulting spectra, however, are too soft to simultaneously fit PAMELA and Fermi LAT. Moreover, the non-observation of any excess in the 

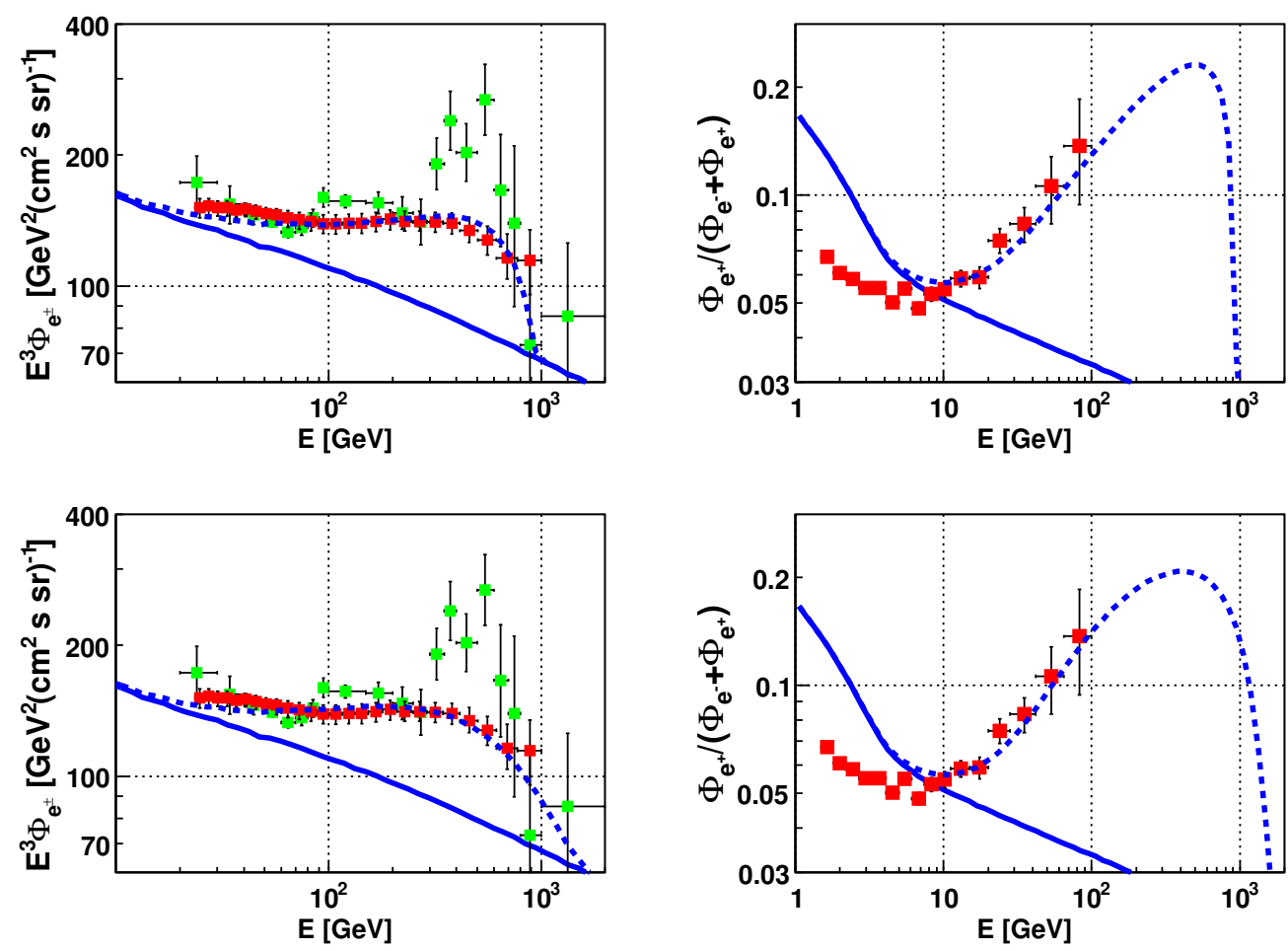

Figure 1: Left panels: fit of $L_{1} L_{3} \bar{E}_{3}$ (top) and $L_{2} L_{3} \bar{E}_{3}$ (bottom) operators (dashed blue) and GALPROP background (solid blue) to electron-plus-positron spectrum from Fermi LAT (red, with error bars). Also shown is ATIC data (green, with error bars). Right panels: data on positron fraction from PAMELA (red, with error bars) shown with GALPROP background (solid blue) and the result of the fit to the Fermi LAT data for the $L_{1} L_{3} \bar{E}_{3}$ and $L_{2} L_{3} \bar{E}_{3}$ operators (dashed blue).

antiproton data reported by PAMELA [12] essentially excludes all attempts with $L Q \bar{D}$ and $\bar{U} \bar{D} \bar{D}$ operators.

We focus therefore on the nine $L L \bar{E}$ operators. When attempting fits with these operators, it becomes clear that the three operators with an SU(2) singlet field of the electron type $\left(\bar{E}_{1}\right)$ give a spectrum that is too hard; in order to get both a sufficiently soft electron-plus-positron spectrum and a large enough positron contribution at PAMELA energies, tau flavour in the SU(2) singlet component, $\bar{E}_{3}$, works better. However, this is only true for single coupling dominance; if combinations of operators are considered, additional good fits can be obtained.

For gravitino masses around $2 \mathrm{TeV}, L_{1} L_{2} \bar{E}_{3}$ and $L_{1} L_{3} \bar{E}_{3}$ give good fits to both PAMELA and Fermi LAT. If the gravitino mass is increased towards $4 \mathrm{TeV}, L_{2} L_{3} \bar{E}_{3}$ also gives a good fit. The absence of electron flavour requires a large gravitino mass in order to reach the high end of the Fermi LAT data. Figure 1 shows two such fits; $L_{1} L_{3} \bar{E}_{3}\left(L_{2} L_{3} \bar{E}_{3}\right)$ with gravitino masses of 1.8 (3.7) $\mathrm{TeV}$, and other sparticle masses set to 2 (6) $\mathrm{TeV}$. 


\section{Gamma Rays}

With a gravitino decay through an $L L \bar{E}$ operator, gamma rays appear in two ways: internal bremsstrahlung off the produced leptons and from the decay of mesons (mostly $\pi^{0}$ ) from $\tau$ decay. Figure 2 shows the resulting gamma ray flux expected for experiments in the solar system. As one can see, the gravitino masses that we consider are too large to be in conflict with the EGRET data on extragalactic diffuse emission, but Fermi LAT will eventually be able to either find some excess, or exclude this model, for the parameter space under consideration.

\section{Conclusions}

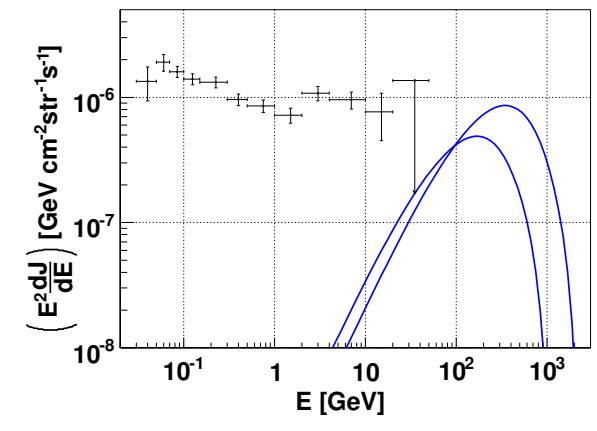

Figure 2: Photon spectra for the fits of the operators $L_{1} L_{3} \bar{E}_{3}$ and $L_{2} L_{3} \bar{E}_{3}$ to the Fermi LAT data. For comparison we also give the EGRET data on extragalactic diffuse emission [13].

Within R-parity violating SUSY models, gravitinos can be natural dark matter candidates. For trilinear R-parity violating operators of the $L L \bar{E}$ type, the recent anomalies in cosmic ray electrons and positrons can be accurately explained without contradicting other cosmic ray measurements. This scenario will be tested in the near future by the diffuse gamma ray data from Fermi LAT.

\section{References}

[1] F. Takayama and M. Yamaguchi, Phys. Lett. B 485 (2000) 388 [hep-ph/ 0005214 ].

[2] N.-E. Bomark, S. Lola, P. Osland and A. R. Raklev, in preparation.

[3] A. Ibarra and D. Tran, JCAP 0807, 002 (2008) [ast ro-ph / 0804 . 4596]; W. Buchmuller, A. Ibarra, T. Shindou, F. Takayama and D. Tran, [arXiv:0906.1187].

[4] N.-E. Bomark, S. Lola, P. Osland and A. R. Raklev, Phys. Lett. B 677 (2009) 62 [hep-ph/0811.2969].

[5] O. Adriani et al. [PAMELA Collaboration], Nature 458 (2009) 607 [astro-ph/ 0810 . 4995].

[6] J. Chang et al., Nature 456 (2008) 362.

[7] A. A. Abdo et al. [The Fermi LAT Collaboration], Phys. Rev. Lett. 102 (2009) 181101 [astro-ph.HE/0905.0025].

[8] D. Grasso et al. [FERMI-LAT Collaboration], astro-ph. HE/0905.0636.

[9] T. Sjostrand, S. Mrenna and P. Skands, JHEP 0605 (2006) 026 [hep-ph / 0603175 ].

[10] A. W. Strong and I. V. Moskalenko, Adv. Space Res. 27 (2001) 717 [astro-ph/0101068].

[11] J. F. Navarro, C. S. Frenk and S. D. M. White, Astrophys. J. 490 (1997) 493 [astro-ph/9611107].

[12] O. Adriani et al. [PAMELA Collaboration], Phys. Rev. Lett. 102 (2009) 051101 [astro-ph/0810.4994].

[13] A. W. Strong, I. V. Moskalenko and O. Reimer, Astrophys. J. 613 (2004) 956 [astro-ph/0405441]. 